\title{
LAGUERRE-GAUSSIAN MODES AND THE WIGNER TRANSFORM
}

\author{
MICHAEL VANVALKENBURGH
}

\begin{abstract}
Recent developments in laser physics have called renewed attention to LaguerreGaussian (LG) beams of paraxial light. In this paper we consider the corresponding LG modes for the two-dimensional harmonic oscillator, which appear in the transversal plane at the laser beam's waist. We see how they arise as Wigner transforms of HermiteGaussian modes, and we proceed to find a closed form for their own Wigner transforms, providing an alternative to the methods of Simon and Agarwal. Our main observation is that the Wigner transform intertwines the creation and annihilation operators for the two classes of modes.
\end{abstract}

Keywords: Laguerre-Gaussian modes, Laguerre-Gaussian beams, Wigner transform, optical angular momentum, orbital angular momentum

\section{INTRODUCTION}

In the early 1990s it was observed that a Laguerre-Gaussian (LG) beam of paraxial light has a well-defined orbital angular momentum and that such a beam may be created from a Hermite-Gaussian (HG) beam by way of an astigmatic optical system [1]. This discovery was soon to find applications, for example, in biology (for "optical tweezers") and in the study of quantum entanglement [2]. Additionally, recent experiments suggest that beams with orbital angular momentum might be generated in situ, in free electron lasers 3 .

In this paper we consider the corresponding LG modes for the two-dimensional harmonic oscillator, which appear in the transversal plane at the laser beam's waist. Indeed, one may use the operator algebra of the harmonic oscillator to study the analytical forms of the HG and LG beams [4], [5]. Here we demonstrate that the LG modes arise as Wigner transforms of HG modes, and we proceed to find their own Wigner transforms. Our methods provide an alternative to those of Gase [6] and those of Simon and Agarwal [7; in particular, Simon and Agarwal were first to discover the closed form expression that we rederive here, using our new point of view.

We define the $d$-dimensional Wigner transform as

$$
W_{d}(f, g)(\vec{x}, \vec{\xi})=(2 \pi)^{-\frac{d}{2}} \int e^{i \vec{p} \cdot \vec{\xi}} \overline{f\left(\frac{\vec{x}+\vec{p}}{\sqrt{2}}\right)} g\left(\frac{\vec{x}-\vec{p}}{\sqrt{2}}\right) d \vec{p}
$$

for functions $f, g$ of $d$ variables. If $d=1$, we omit the subscript. The Wigner transform is often restricted to the case when $f \equiv g$, and then one writes $W_{d}(f):=W_{d}(f, f)$. More generally, we define the extended Wigner transform, of a function $F$ of $2 d$ variables, as

$$
\tilde{W}_{d}(F)(\vec{x}, \vec{\xi})=(2 \pi)^{-\frac{d}{2}} \int e^{i \vec{p} \cdot \vec{\xi}} F\left(\frac{\vec{x}+\vec{p}}{\sqrt{2}}, \frac{\vec{x}-\vec{p}}{\sqrt{2}}\right) d \vec{p} .
$$


This more general transform is fundamental for the study of LG modes; this is our main insight and provides the central theme for our paper.

Our main results are summarized in the following theorem.

Theorem 1. Let $h_{j}$ denote the $j^{\text {th }}$ Hermite function, so that $h_{j k}(x, y)=h_{j}(x) h_{k}(y)$ are the HG modes.

(a) The extended Wigner transform intertwines the creation and annihilation operators of the $H G$ and $L G$ modes.

(b) The $L G$ modes are precisely $\tilde{W}\left(h_{j k}\right)$ : the extended Wigner transforms of the $H G$ modes.

(c) The Wigner transforms of the $L G$ modes are given by

$$
\begin{aligned}
W_{2}\left(\tilde{W}\left(h_{j k}\right), \tilde{W}\left(h_{m n}\right)\right)(\vec{x}, \vec{\xi}) \\
=\tilde{W}\left(h_{j m}\right)\left(\frac{x_{1}+\xi_{2}}{\sqrt{2}}, \frac{\xi_{1}-x_{2}}{\sqrt{2}}\right) \tilde{W}\left(h_{k n}\right)\left(\frac{x_{1}-\xi_{2}}{\sqrt{2}}, \frac{\xi_{1}+x_{2}}{\sqrt{2}}\right) .
\end{aligned}
$$

We remark that part (b) of the theorem follows from part (a) and the fact that $\tilde{W}\left(h_{00}\right)=$ $h_{00}$.

In the case $(j, k)=(m, n)$, the formula (1) was proven by Simon and Agarwal, using the metaplectic representation, and in fact their proof extends to the general case without modification [7]. The generalization should be useful in light of the relationship between the Wigner transform and the Weyl quantization of observables. Indeed, for any tempered distribution $\sigma \in \mathcal{S}^{\prime}\left(\mathbb{R}^{2 d}\right)$ we may define the Weyl quantization of $\sigma$ as the [pseudodifferential] operator given by

$$
\left(\mathrm{Op}^{W}(\sigma) u\right)(x)=2^{-\frac{3 d}{2}} \pi^{-d} \iint \exp \left(\frac{i(x-y) \xi}{\sqrt{2}}\right) \sigma\left(\frac{x+y}{\sqrt{2}}, \xi\right) u(y) d y d \xi .
$$

The normalization factor is chosen so that $\mathrm{Op}^{W}(1)$ is the identity operator. Then it is easy to check that for any Schwartz functions $f, g \in \mathcal{S}\left(\mathbb{R}^{d}\right)$ we have

$$
\left\langle f \mid \mathrm{Op}^{W}(\sigma) g\right\rangle=2^{-d} \pi^{-\frac{d}{2}} \iint \sigma(x, \xi) W_{d}(f, g)(x, \xi) d x d \xi .
$$

For an exposition of the Weyl calculus of pseudodifferential operators, the reader may consult the book of Gerald Folland [8].

In Section 2 we review the theory of the one-dimensional harmonic oscillator. Since this subject is very well known, we will be brief, so the section is mostly a means of setting up notation for the following sections. In Section 3, we review the theory of the two-dimensional harmonic oscillator. Our primary reference for Sections 2 and 3 is the classic book of Messiah [9].

In Section 4 we write the $\{Q\}$-representation in terms of complex variables, which provides a clean expression for the LG modes in terms of Laguerre polynomials. The connection with LG modes of paraxial light is briefly exhibited in Section 5 .

The LG modes are expressed as Wigner transforms in Section 6, and in Section 7 we calculate their own Wigner transforms. 


\section{The One-Dimensional Harmonic Oscillator}

We begin by setting up the notation for the one-dimensional harmonic oscillator, given by

$$
H=\frac{1}{2}\left(P^{2}+Q^{2}\right), \quad \text { where }[Q, P]=i .
$$

We set

$$
a=\frac{1}{\sqrt{2}}(Q+i P) \quad \text { and } \quad a^{\dagger}=\frac{1}{\sqrt{2}}(Q-i P)
$$

so that

$$
\left[a, a^{\dagger}\right]=1 \quad \text { and } \quad H=\frac{1}{2}\left(a a^{\dagger}+a^{\dagger} a\right)
$$

Moreover, we define

$$
N=a^{\dagger} a
$$

so that

$$
H=N+\frac{1}{2} \text {. }
$$

It is well known that $\operatorname{Spec}(N)=\{0,1,2, \ldots\}$ consists of nondegenerate eigenvalues and that $a^{\dagger}$ and $a$ are the "ladder operators": the creation and annihilation operators, respectively. The associated eigenvectors form a complete set, so we can normalize to get an orthonormal basis of eigenvectors for the observable $N$ :

$$
|0\rangle, \quad|1\rangle, \quad|2\rangle, \ldots
$$

corresponding to the eigenvalues

$$
0, \quad 1, \quad 2, \ldots
$$

In the $\{Q\}$-Representation (that is, the Schrödinger representation where states are considered as functions of position) the ladder operators are written as

$$
a=\frac{1}{\sqrt{2}}\left(x+\frac{d}{d x}\right)
$$

and

$$
a^{\dagger}=\frac{1}{\sqrt{2}}\left(x-\frac{d}{d x}\right) \text {. }
$$

The ground state $h_{0}$ satisfies

$$
\left[\frac{d}{d x}+x\right] h_{0}(x)=0
$$

so that

$$
h_{0}(x)=\pi^{-\frac{1}{4}} e^{-\frac{1}{2} x^{2}} .
$$

Using the ladder operators, we get the rest of the eigenvectors, which are precisely the Hermite functions:

$$
\begin{aligned}
h_{n}(x) & =\pi^{-\frac{1}{4}}(n !)^{-\frac{1}{2}} 2^{-\frac{n}{2}}\left(x-\frac{d}{d x}\right)^{n} e^{-\frac{1}{2} x^{2}} \\
& =\pi^{-\frac{1}{4}}(n !)^{-\frac{1}{2}} 2^{-\frac{n}{2}}(-1)^{n} e^{\frac{1}{2} x^{2}} \frac{d^{n}}{d x^{n}} e^{-x^{2}} \\
& =\pi^{-\frac{1}{4}}(n !)^{-\frac{1}{2}} 2^{-\frac{n}{2}} e^{-\frac{1}{2} x^{2}} H_{n}(x)
\end{aligned}
$$


where the $H_{n}$ are the Hermite polynomials

$$
H_{n}(x)=(-1)^{n} e^{x^{2}} \frac{d^{n}}{d x^{n}} e^{-x^{2}}
$$

\section{The Two-Dimensional Isotropic Harmonic Oscillator}

In two dimensions, we have

$$
H=\frac{1}{2}\left(P_{1}^{2}+P_{2}^{2}+Q_{1}^{2}+Q_{2}^{2}\right)
$$

with creation and annihilation operators inherited from the one-dimensional case:

$$
\begin{aligned}
& a_{j}^{\dagger}=\frac{1}{\sqrt{2}}\left(Q_{j}-i P_{j}\right), \\
& a_{j}=\frac{1}{\sqrt{2}}\left(Q_{j}+i P_{j}\right), \quad j=1,2 .
\end{aligned}
$$

These are interpreted as creation and annihilation operators, respectively, of quanta of type $j \in\{1,2\}$. They satisfy

$$
\begin{gathered}
{\left[a_{i}, a_{j}\right]=\left[a_{i}^{\dagger}, a_{j}^{\dagger}\right]=0,} \\
{\left[a_{i}, a_{j}^{\dagger}\right]=\delta_{i j} .}
\end{gathered}
$$

And we have the corresponding "number operators"

$$
N_{j}=a_{j}^{\dagger} a_{j}, \quad j=1,2,
$$

with

$$
N=N_{1}+N_{2}
$$

representing the total number.

Now the eigenvalues for

$$
H=N+1
$$

are

$$
\{1,2,3, \ldots\}
$$

and the eigenvalue $j$ has degeneracy $j$. Hence there are many possible choices of eigenbasis. One often simply takes the basis consisting of tensor products of the one-dimensional eigenvectors, corresponding to the complete set of commuting observables $\left\{N_{1}, N_{2}\right\}$. The elements of this basis are given by

$$
\left|n_{1} n_{2}\right\rangle=\left(n_{1} ! n_{2} !\right)^{-\frac{1}{2}} a_{1}^{\dagger n_{1}} a_{2}^{\dagger n_{2}}|00\rangle .
$$

These are precisely the HG modes; in the Schrödinger representation, they are tensor products of Hermite functions.

But here we will construct another basis.

The angular momentum operator $L$ is defined by

$$
L=Q_{1} P_{2}-Q_{2} P_{1}=i\left(a_{1} a_{2}^{\dagger}-a_{1}^{\dagger} a_{2}\right),
$$

and one may check that it is a constant of motion. We will show that $\{N, L\}$ is another complete set of commuting observables. 
Let

$$
A_{ \pm}=\frac{1}{\sqrt{2}}\left(a_{1} \mp i a_{2}\right)
$$

and

$$
A_{ \pm}^{\dagger}=\frac{1}{\sqrt{2}}\left(a_{1}^{\dagger} \pm i a_{2}^{\dagger}\right)
$$

Then

$$
\begin{gathered}
{\left[A_{r}, A_{s}\right]=\left[A_{r}^{\dagger}, A_{s}^{\dagger}\right]=0 \quad \text { and }} \\
{\left[A_{r}, A_{s}^{\dagger}\right]=\delta_{r s} \quad \text { for } r, s \in\{+,-\} .}
\end{gathered}
$$

We think of $A_{r}^{\dagger}, A_{r}$ as creation and annihilation operators, respectively, of quanta of type $r \in\{+,-\}$, so then

$$
N_{r}=A_{r}^{\dagger} A_{r}
$$

represents the number of " $r$ quanta".

Hence the problem of finding eigenvectors common to $N_{+}$and $N_{-}$is formally equivalent to finding eigenvectors common to $N_{1}$ and $N_{2}$. So by the usual arguments we see that

$$
\operatorname{Spec}\left(N_{+}\right)=\operatorname{Spec}\left(N_{-}\right)=\{0,1,2, \ldots\}
$$

and that these two observables, $N_{+}$and $N_{-}$, form a complete set of commuting observables: to each $\left(n_{+}, n_{-}\right)$there is a common eigenvector, denoted by $\left|n_{+} n_{-}\right\rangle$, that is unique to within a constant.

In fact,

$$
A_{+}|00\rangle=A_{-}|00\rangle=0
$$

and the states

$$
\left|n_{+} n_{-}\right\rangle=\left(n_{+} ! n_{-} !\right)^{-\frac{1}{2}} A_{+}^{\dagger n_{+}} A_{-}^{\dagger n_{-}}|00\rangle
$$

form a complete orthonormal eigenbasis common to $N_{+}$and $N_{-}$:

$$
N_{+}\left|n_{+} n_{-}\right\rangle=n_{+}\left|n_{+} n_{-}\right\rangle
$$

and

$$
N_{-}\left|n_{+} n_{-}\right\rangle=n_{-}\left|n_{+} n_{-}\right\rangle \text {. }
$$

We find that

$$
N=N_{+}+N_{-}
$$

and that

$$
L=N_{+}-N_{-} .
$$

Hence $N$ and $L$ form a complete set of commuting observables. Moreover,

$$
\left[L, A_{ \pm}^{\dagger}\right]= \pm A_{ \pm}^{\dagger}
$$

and

$$
\left[L, A_{ \pm}\right]=\mp A_{ \pm}
$$

so when they act upon an eigenvector of $L, A_{+}^{\dagger}$ and $A_{-}$increase $L$ by one unit, and $A_{-}^{\dagger}$ and $A_{+}$decrease $L$ by one unit. So it is natural to consider $N_{+}$as the number of particles with positive charge, $N_{-}$as the number of particles with negative charge, and $L$ as the total charge (to within a constant). 


\section{The Two-Dimensional Isotropic Harmonic Oscillator in the $\{Q\}$-REPRESENTATION}

In the $\{Q\}$-representation, we write

$$
\begin{array}{ll}
a_{1}=\frac{1}{\sqrt{2}}\left(x+\frac{\partial}{\partial x}\right), & a_{2}=\frac{1}{\sqrt{2}}\left(y+\frac{\partial}{\partial y}\right), \\
a_{1}^{\dagger}=\frac{1}{\sqrt{2}}\left(x-\frac{\partial}{\partial x}\right), & a_{2}^{\dagger}=\frac{1}{\sqrt{2}}\left(y-\frac{\partial}{\partial y}\right),
\end{array}
$$

so that we have

$$
\begin{aligned}
& A_{+}=\frac{1}{2}\left(x+\frac{\partial}{\partial x}-i\left(y+\frac{\partial}{\partial y}\right)\right), \\
& A_{-}=\frac{1}{2}\left(x+\frac{\partial}{\partial x}+i\left(y+\frac{\partial}{\partial y}\right)\right), \\
& A_{+}^{\dagger}=\frac{1}{2}\left(x-\frac{\partial}{\partial x}+i\left(y-\frac{\partial}{\partial y}\right)\right), \\
& A_{-}^{\dagger}=\frac{1}{2}\left(x-\frac{\partial}{\partial x}-i\left(y-\frac{\partial}{\partial y}\right)\right) .
\end{aligned}
$$

In complex notation, these take a simple form. We write $z=x+i y$, so that

$$
\frac{\partial}{\partial z}=\frac{1}{2}\left(\frac{\partial}{\partial x}+\frac{1}{i} \frac{\partial}{\partial y}\right) \quad \text { and } \quad \frac{\partial}{\partial \bar{z}}=\frac{1}{2}\left(\frac{\partial}{\partial x}-\frac{1}{i} \frac{\partial}{\partial y}\right) .
$$

Then

$$
\begin{array}{rlrl}
A_{+} & =\frac{1}{2} \bar{z}+\frac{\partial}{\partial z}, & A_{-} & =\frac{1}{2} z+\frac{\partial}{\partial \bar{z}}, \\
A_{+}^{\dagger}=\frac{1}{2} z-\frac{\partial}{\partial \bar{z}}, & A_{-}^{\dagger}=\frac{1}{2} \bar{z}-\frac{\partial}{\partial z} .
\end{array}
$$

Moreover, we can write

$$
A_{+}^{\dagger}=-e^{\frac{1}{2} z \bar{z}} \frac{\partial}{\partial \bar{z}} e^{-\frac{1}{2} z \bar{z}} \quad \text { and } \quad A_{-}^{\dagger}=-e^{\frac{1}{2} z \bar{z}} \frac{\partial}{\partial z} e^{-\frac{1}{2} z \bar{z}} .
$$

The ground state is given by

$$
u_{0}(z, \bar{z})=\pi^{-\frac{1}{2}} e^{-\frac{1}{2} z \bar{z}} \equiv\langle z, \bar{z} \mid 00\rangle,
$$

and of course we get all other eigenvectors by applying the creation operators to this. These are precisely the LG modes. Explicitly,

$$
\begin{aligned}
\left\langle z, \bar{z} \mid n_{+} n_{-}\right\rangle & =\left\langle z, \bar{z}\left|\left(n_{+} ! n_{-} !\right)^{-\frac{1}{2}} A_{+}^{\dagger n_{+}} A_{-}^{\dagger n_{-}}\right| 00\right\rangle \\
& =\pi^{-\frac{1}{2}}\left(n_{+} ! n_{-} !\right)^{-\frac{1}{2}}(-1)^{n_{+}+n_{-}} e^{\frac{1}{2} z \bar{z}}\left(\frac{\partial}{\partial \bar{z}}\right)^{n_{+}}\left(\frac{\partial}{\partial z}\right)^{n_{-}} e^{-z \bar{z}}
\end{aligned}
$$

We will now formulate this in terms of Laguerre polynomials. 
First suppose that $n_{+} \geq n_{-}$. Then

$$
\begin{aligned}
\left\langle z, \bar{z} \mid n_{+} n_{-}\right\rangle & =\pi^{-\frac{1}{2}}\left(n_{+} ! n_{-} !\right)^{-\frac{1}{2}}(-1)^{n_{+}+n_{-}} e^{\frac{1}{2} z \bar{z}}\left(\frac{\partial}{\partial z}\right)^{n_{-}}\left[(-z)^{n_{+}} e^{-z \bar{z}}\right] \\
& =\pi^{-\frac{1}{2}}\left(n_{+} ! n_{-} !\right)^{-\frac{1}{2}}(-1)^{n_{-}} e^{\frac{1}{2} z \bar{z}} \bar{z}^{\left(n_{-}-n_{+}\right)}\left(\frac{\partial}{\partial(z \bar{z})}\right)^{n_{-}}\left[(z \bar{z})^{n_{+}} e^{-z \bar{z}}\right] .
\end{aligned}
$$

We define the Laguerre polynomials by

$$
L_{n}^{\alpha}(x)=\frac{x^{-\alpha} e^{x}}{n !} \frac{d^{n}}{d x^{n}}\left(e^{-x} x^{n+\alpha}\right)
$$

for $n \geq 0$ and $\alpha>-1$. Then we see that, when $n_{+} \geq n_{-}$,

$$
\left\langle z, \bar{z} \mid n_{+} n_{-}\right\rangle=\pi^{-\frac{1}{2}}\left(\frac{n_{-} !}{n_{+} !}\right)^{\frac{1}{2}}(-1)^{n_{-}} z^{\left(n_{+}-n_{-}\right)} e^{-\frac{1}{2} z \bar{z}} L_{n_{-}}^{n_{+}-n_{-}}(z \bar{z}) .
$$

On the other hand, if $n_{+} \leq n_{-}$, similar arguments show that

$$
\left\langle z, \bar{z} \mid n_{+} n_{-}\right\rangle=\pi^{-\frac{1}{2}}\left(\frac{n_{+} !}{n_{-} !}\right)^{\frac{1}{2}}(-1)^{n_{+}} \bar{z}^{\left(n_{-}-n_{+}\right)} e^{-\frac{1}{2} z \bar{z}} L_{n_{+}}^{n_{-}-n_{+}}(z \bar{z}) .
$$

We summarize this in the following theorem:

Theorem 2. We consider the states $\left|n_{+} n_{-}\right\rangle$in the $\{Q\}$-representation with position coordinates $(x, y) \in \mathbb{R}^{2}$, and we write $z=x+i y$. Then

$$
\left\langle z, \bar{z} \mid n_{+} n_{-}\right\rangle= \begin{cases}\pi^{-\frac{1}{2}}\left(\frac{n_{-} !}{n_{+} !}\right)^{\frac{1}{2}}(-1)^{n_{-}} z^{\left(n_{+}-n_{-}\right)} e^{-\frac{1}{2} z \bar{z}} L_{n_{-}}^{n_{+}-n_{-}}(z \bar{z}) & \text { if } n_{+} \geq n_{-}, \text {and } \\ \pi^{-\frac{1}{2}}\left(\frac{n_{+} !}{n_{-} !}\right)^{\frac{1}{2}}(-1)^{n_{+}} \bar{z}^{\left(n_{-}-n_{+}\right)} e^{-\frac{1}{2} z \bar{z}} L_{n_{+}}^{n_{-} n_{+}}(z \bar{z}) & \text { if } n_{+} \leq n_{-} .\end{cases}
$$

\section{Comparison With LG Modes of Paraxial Light}

The time-harmonic field amplitudes of LG optical modes are expressed in cylindrical coordinates, with propagation coordinate $z$ along the beam axis, as

$$
\tilde{u}_{p, \ell}(\mathbf{r}) \propto e^{-i \Phi(\mathbf{r})} e^{-\frac{r^{2}}{w(z)^{2}}}\left(\frac{r \sqrt{2}}{w(z)}\right)^{|\ell|} L_{p}^{|\ell|}\left(\frac{2 r^{2}}{w(z)^{2}}\right)
$$

where the phase is

$$
\Phi(\mathbf{r})=\ell \phi-k z+\frac{k r^{2}}{2 R(z)}-(2 p+\ell+1) \Psi(z)
$$

and where again $L_{p}^{|\ell|}$ is the Laguerre polynomial with radial and azimuthal indices $p$ and $\ell$, respectively. The term $\Psi(z)=\tan ^{-1}\left(\frac{z}{z_{R}}\right)$ is part of the Gouy phase, $R(z)=\frac{z_{R}^{2}+z^{2}}{z}$ is the radius of curvature, $w(z)=w_{0} \sqrt{1+\left(\frac{z}{z_{R}}\right)^{2}}$ is the beam waist, and $z_{R}=\frac{1}{2} k w_{0}^{2}$ is the Rayleigh range. We see that the total phase evolves helically along the propagation coordinate $z$. For more on this, see, for example, [2], [3], [10]. 
In the transversal plane at the beam waist $(\mathrm{z}=0)$, we get

$$
\tilde{u}_{p, \ell}(\mathbf{r}) \propto e^{-i \ell \phi} e^{-\frac{r^{2}}{w_{0}^{2}}}\left(\frac{r \sqrt{2}}{w_{0}}\right)^{|\ell|} L_{p}^{|\ell|}\left(\frac{2 r^{2}}{w_{0}^{2}}\right),
$$

which is essentially what we have in Theorem 2 .

\section{LG Modes Are Wigner Transforms}

Physicists in the optics community recently computed the Wigner transforms of LG modes [6], [7]. Surprisingly, the states $\left|n_{+} n_{-}\right\rangle$are already themselves Wigner transforms of Hermite functions. The Hermite functions, as in Section 2, are given by

$$
h_{j}(x)=\pi^{-\frac{1}{4}}(j !)^{-\frac{1}{2}} 2^{-\frac{j}{2}}(-1)^{j} e^{\frac{1}{2} x^{2}} \frac{d^{j}}{d x^{j}} e^{-x^{2}},
$$

which, as we know, satisfy the orthogonality relations

$$
\left\langle h_{j} \mid h_{k}\right\rangle=\delta_{j k}
$$

And we continue to write the HG modes as

$$
h_{j k}(x, y)=h_{j}(x) h_{k}(y) .
$$

Now, for reference, we list the standard properties of the Wigner transform:

$$
\begin{gathered}
W(f, g)=\overline{W(g, f)} \\
\int W(f, g)(x, \xi) d \xi=\sqrt{2 \pi} f\left(\frac{x}{\sqrt{2}}\right) g\left(\frac{x}{\sqrt{2}}\right), \\
\iint W(f, g)(x, \xi) d x d \xi=2 \sqrt{\pi}\langle f \mid g\rangle, \\
\int W(f, g)(x, \xi) d x=\sqrt{2 \pi \hat{f}\left(\frac{\xi}{\sqrt{2}}\right)} \hat{g}\left(\frac{\xi}{\sqrt{2}}\right),
\end{gathered}
$$

where $\hat{f}(\xi)=\frac{1}{\sqrt{2 \pi}} \int e^{-i x \xi} f(x) d x$ is the Fourier transform, and ("Moyal's identity")

$$
\left\langle W\left(f_{1}, g_{1}\right) \mid W\left(f_{2}, g_{2}\right)\right\rangle=\overline{\left\langle f_{1} \mid f_{2}\right\rangle}\left\langle g_{1} \mid g_{2}\right\rangle .
$$

These properties are well known and are easily checked. But there is another elementary property which seems to be a new observation:

Theorem 3. The extended Wigner transform intertwines the creation operators of the $H G$ and $L G$ modes, and it intertwines the annihilation operators of the $H G$ and $L G$ modes:

$$
\begin{array}{lll}
A_{+}^{\dagger} \tilde{W}=\tilde{W} a_{1}^{\dagger}, & A_{-}^{\dagger} \tilde{W}=\tilde{W} a_{2}^{\dagger}, \\
A_{+} \tilde{W}=\tilde{W} a_{1}, & A_{-} \tilde{W}=\tilde{W} a_{2} .
\end{array}
$$


The proof is a direct calculation, using the expressions (2) and (3). As a first application, it is now easy to see that the extended Wigner transform commutes with the operators $H$ and $N$.

Additionally using the fact that $\tilde{W}\left(h_{00}\right)=h_{00}$, we have the following corollary:

Corollary 1. We consider the $L G$ modes $\left|n_{+} n_{-}\right\rangle$in the $\{Q\}$-representation with position coordinates $(x, y) \in \mathbb{R}^{2}$. Then we have

$$
\left\langle x, y \mid n_{+} n_{-}\right\rangle=W\left(h_{n_{+}}, h_{n_{-}}\right)(x, y) .
$$

Combining this with Theorem 2, we have a formula:

Corollary 2. Suppose $x, y \in \mathbb{R}$, and let $z=x+i y$. Then

$$
W\left(h_{j}, h_{k}\right)(x, y)= \begin{cases}\pi^{-\frac{1}{2}}\left(\frac{k !}{j !}\right)^{\frac{1}{2}}(-1)^{k} z^{j-k} e^{-\frac{1}{2} z \bar{z}} L_{k}^{j-k}(z \bar{z}) & \text { if } j \geq k, \text { and } \\ \pi^{-\frac{1}{2}}\left(\frac{j !}{k !}\right)^{\frac{1}{2}}(-1)^{j} \bar{z}^{k-j} e^{-\frac{1}{2} z \bar{z}} L_{j}^{k-j}(z \bar{z}) & \text { if } j \leq k .\end{cases}
$$

Corollary 2 by itself is not new. For example, two independent proofs may be found in Folland's book [8], although we caution the reader that his statement of the result contains a misprint.

So we have yet another way of understanding the orthogonality relations of the states $\left|n_{+} n_{-}\right\rangle$: as being inherited from the orthogonality of the Hermite functions. Explicitly,

$$
\begin{aligned}
\left\langle m_{+} m_{-} \mid n_{+} n_{-}\right\rangle & =\left\langle W\left(h_{m_{+}}, h_{m_{-}}\right) \mid W\left(h_{n_{+}}, h_{n_{-}}\right)\right\rangle \\
& =\left\langle h_{m_{+}} \mid h_{n_{+}}\right\rangle\left\langle h_{m_{-}} \mid h_{n_{-}}\right\rangle \\
& =\delta_{m_{+} n_{+}} \delta_{m_{-} n_{-}} .
\end{aligned}
$$

As another application of Corollary 1, we can think of the LG modes $\left|n_{+} n_{-}\right\rangle$as being "interference terms" resulting from the quadratic nature of the Wigner transform. If we apply the Wigner transform to sums of Hermite functions, we get interference terms that are LG modes with nonzero angular momentum. In fact, we have the following polarization identity:

$$
\begin{aligned}
\left\langle x, y \mid n_{+} n_{-}\right\rangle=\frac{1}{4} W( & \left.h_{n_{+}}+h_{n_{-}}\right)(x, y)-\frac{1}{4} W\left(h_{n_{+}}-h_{n_{-}}\right)(x, y) \\
& +\frac{i}{4} W\left(h_{n_{+}}-i h_{n_{-}}\right)(x, y)-\frac{i}{4} W\left(h_{n_{+}}+i h_{n_{-}}\right)(x, y) .
\end{aligned}
$$

One may think of the Wigner transform as being a kind of "product". Just as Hermite functions give rise to two-dimensional HG modes, via the tensor product, Hermite functions also give rise to two-dimensional LG modes, via the Wigner transform. To complete this series of relationships, Simon and Agarwal [7] noted that the two-dimensional HG 
and LG modes are unitarily related by the operator

$$
\exp \left(\frac{i \pi}{4} \hat{T}_{1}\right), \quad \text { where } \hat{T}_{1}=x y-\frac{\partial^{2}}{\partial x \partial y} .
$$

Alternatively, we can use the Wigner transform to again show that the HG and LG modes are unitarily related. For functions $F$ of two variables, we consider the extended Wigner transform of $F$ :

$$
\tilde{W} F(x, y)=\frac{1}{\sqrt{2 \pi}} \int e^{i p y} F\left(\frac{x+p}{\sqrt{2}}, \frac{x-p}{\sqrt{2}}\right) d p .
$$

If we write the $\frac{\pi}{4}$-rotation operator as

$$
\begin{aligned}
\left(R_{\frac{\pi}{4}}^{*} F\right)(x, p) & =F\left(R_{\frac{\pi}{4}}(x, p)\right) \\
& =F\left(\frac{x-p}{\sqrt{2}}, \frac{x+p}{\sqrt{2}}\right),
\end{aligned}
$$

and if we write the partial Fourier transform as

$$
\left(\mathcal{F}_{2} F\right)(x, y)=\frac{1}{\sqrt{2 \pi}} \int e^{-i p y} F(x, p) d p,
$$

then we may write the extended Wigner transform as

$$
\tilde{W} F(x, y)=\left(\mathcal{F}_{2} R_{\frac{\pi}{4}}^{*} F\right)(x, y) \text {. }
$$

This is clearly a unitary operator, so, in particular, we again see that the HG modes $h_{j k}$ are unitarily related to the LG modes $\tilde{W}\left(h_{j k}\right)$.

The results of this section are surprising, despite their mathematical simplicity. We are accustomed to thinking of the Wigner transform of a function as being a phase space representation of the function, but here the relevant Wigner transform is actually a function of the position vector $(x, y) \in \mathbb{R}^{2}$, as it is an LG mode. So if we use the Wigner transform to study the phase space properties of LG modes, we are really taking Wigner transforms of Wigner transforms. Gase [6], followed by Simon and Agarwal [7, already recently derived expressions for Wigner transforms of LG modes (Gase in terms of a quadruple sum, and Simon and Agarwal in terms of a closed form involving no summation at all). We will do the same in the next section, but now using our new point of view.

\section{Wigner Transforms of LG Modes}

We now proceed to compute the Wigner transforms of the LG modes $\tilde{W}\left(h_{j k}\right)$. In light of (9), the first step is to consider the action of the Wigner transform on partial Fourier transforms.

\section{Lemma 1.}

$$
W_{2}\left(\mathcal{F}_{2} F, \mathcal{F}_{2} G\right)(\vec{x}, \vec{\xi})=\frac{1}{2 \pi} \iint e^{i \xi_{1} s-i x_{2} t} F\left(\frac{x_{1}+s}{\sqrt{2}}, \frac{-\xi_{2}-t}{\sqrt{2}}\right) G\left(\frac{x_{1}-s}{\sqrt{2}}, \frac{-\xi_{2}+t}{\sqrt{2}}\right) d s d t .
$$


This lemma follows from a direct calculation.

We now apply the lemma to

$$
F=R_{\frac{\pi}{4}}^{*} h_{j k} \quad \text { and } \quad G=R_{\frac{\pi}{4}}^{*} h_{m n} .
$$

That is,

$$
F(\vec{x})=h_{j}\left(\frac{x_{1}-x_{2}}{\sqrt{2}}\right) h_{k}\left(\frac{x_{1}+x_{2}}{\sqrt{2}}\right)
$$

and similarly for $G$. Hence the Wigner transforms of the LG modes are given by

$$
\begin{aligned}
W_{2}\left(\tilde{W}\left(h_{j k}\right), \tilde{W}\left(h_{m n}\right)\right)(\vec{x}, \vec{\xi}) & \\
=\frac{1}{2 \pi} \iint e^{i \xi_{1} s-i x_{2} t} h_{j} & \left(\frac{\left(x_{1}+s\right)+\left(\xi_{2}+t\right)}{2}\right) h_{k}\left(\frac{\left(x_{1}+s\right)-\left(\xi_{2}+t\right)}{2}\right) \\
& h_{m}\left(\frac{\left(x_{1}-s\right)+\left(\xi_{2}-t\right)}{2}\right) h_{n}\left(\frac{\left(x_{1}-s\right)-\left(\xi_{2}-t\right)}{2}\right) d s d t .
\end{aligned}
$$

We may simplify this integral by again rotating the variables by $\frac{\pi}{4}$. We state the result as a theorem.

Theorem 4. The Wigner transforms of the $L G$ modes are products of $L G$ modes, given by the formula

$$
\begin{aligned}
& W_{2}\left(\tilde{W}\left(h_{j k}\right), \tilde{W}\left(h_{m n}\right)\right)(\vec{x}, \vec{\xi}) \\
& =\tilde{W}\left(h_{j m}\right)\left(\frac{x_{1}+\xi_{2}}{\sqrt{2}}, \frac{\xi_{1}-x_{2}}{\sqrt{2}}\right) \tilde{W}\left(h_{k n}\right)\left(\frac{x_{1}-\xi_{2}}{\sqrt{2}}, \frac{\xi_{1}+x_{2}}{\sqrt{2}}\right) .
\end{aligned}
$$

In the special case $(j, k)=(m, n)$, we have the following formula of Simon and Agarwal [7. It is now proven by combining Corollary 2 with Theorem 4 .

Corollary 3. For $(\vec{x}, \vec{\xi})=\left(x_{1}, x_{2}, \xi_{1}, \xi_{2}\right) \in \mathbb{R}^{2} \times \mathbb{R}^{2}$, we let

$$
Q_{0}=\frac{1}{2}\left(|\vec{x}|^{2}+|\vec{\xi}|^{2}\right) \quad \text { and } \quad Q_{2}=\left(x_{1} \xi_{2}-x_{2} \xi_{1}\right) .
$$

Then we have

$$
W_{2}\left(\tilde{W}\left(h_{j k}\right), \tilde{W}\left(h_{j k}\right)\right)(\vec{x}, \vec{\xi})=\pi^{-1}(-1)^{j+k} e^{-Q_{0}} L_{j}^{0}\left(Q_{0}+Q_{2}\right) L_{k}^{0}\left(Q_{0}-Q_{2}\right) .
$$

For comparison, we also give the Wigner transforms of HG modes:

$$
W_{2}\left(h_{j k}, h_{m n}\right)(\vec{x}, \vec{\xi})=\tilde{W}\left(h_{j m}\right)\left(x_{1}, \xi_{1}\right) \tilde{W}\left(h_{k n}\right)\left(x_{2}, \xi_{2}\right) .
$$

In the particular case $(j, k)=(m, n)$, if we let

$$
Q_{3}=\frac{1}{2}\left(x_{1}^{2}-x_{2}^{2}+\xi_{1}^{2}-\xi_{2}^{2}\right)
$$

then we have the formula

$$
W_{2}\left(h_{j k}, h_{j k}\right)(\vec{x}, \vec{\xi})=\pi^{-1}(-1)^{j+k} e^{-Q_{0}} L_{j}^{0}\left(Q_{0}+Q_{3}\right) L_{k}^{0}\left(Q_{0}-Q_{3}\right) .
$$


Acknowledgements: The author's interest in this topic is due to a fascinating talk given by J. B. Rosenzweig, entitled "Accelerator Physics: New Light Sources and Medical Imaging Techniques". The author also wishes to thank M. Hitrik and R. Simon for their helpful comments.

\section{REFERENCES}

[1] L. Allen, M. W. Beijersbergen, R. J. C. Spreeuw, and J. P. Woerdman, Phys. Rev. A 45, 8185 (1992).

[2] L. Allen, S. M. Barnett, and M. J. Padgett, Optical Angular Momentum (IOP Publishing, Bristol, UK, 2003).

[3] E. Hemsing et al., in Proceedings of the 2007 Particle Accelerator Conference (IEEE, Albuquerque, NM, 2007), p.1263; http://www.jacow.org.

[4] G. Nienhuis and L. Allen, Phys. Rev. A 48, 656 (1993).

[5] J. Visser and G. Nienhuis, Phys. Rev. A 70, 013809 (2004).

[6] R. Gase, IEEE J. Quantum Electron. 31, 1811 (1995).

[7] R. Simon and G. S. Agarwal, Opt. Lett. 25, 1313 (2000).

[8] G. B. Folland, Harmonic Analysis in Phase Space (Annals of Mathematics Studies, 122, Princeton University Press, Princeton, NJ, 1989).

[9] A. Messiah, Quantum Mechanics (John Wiley \& Sons, New York, NY, 1958).

[10] A. E. Siegman, Lasers (University Science Books, Mill Valley, CA, 1986).

UCLA Department of Mathematics, Los Angeles, CA 90095-1555, USA

E-mail address: mvanvalk@ucla.edu 